\title{
Implementación de herramientas tecnológicas en los procesos de enseñanza-aprendizaje de los triángulos
}

Implementation of technological tools in the teaching processes- learning of the triangles 


\title{
Implementación de herramientas tecnológicas en los procesos de enseñanza-aprendizaje de los triángulos ${ }^{1}$
}

\section{Implementation of technological tools in the teaching processes- learning of the triangles}

\author{
Fredys A. Simanca H. ${ }^{2}$, Alexandra Abuchar Porras ${ }^{3}$, Fabián Blanco Garrido4, \\ Pablo Carreño Hernández ${ }^{5}$ \\ 2,4,5Universidad Cooperativa de Colombia, Colombia. ${ }^{3}$ Universidad Distrital Francisco José de Caldas
}

Artículo recibido en enero de 2017; artículo aceptado en abril de 2017

Citación del artículo: Simanca, F., Abuchar, A., Blanco, F. \& Carreño, P. (2017). Implementación de herramientas tecnológicas en los procesos de enseñanza-aprendizaje de los triángulos. I+D Revista de Investigaciones, 10(2), julio - diciembre de 2017 , pp. 71 - 79

\begin{abstract}
Resumen
Este trabajo nace de la práctica docente de un equipo de trabajo de conformado por tres instituciones educativas tras identificar la necesidad de potencializar los procesos de enseñanza aprendizaje aplicando las TIC, mediante el desarrollo de herramientas tecnológicas como apoyo a la práctica docente. Para este caso específico, en el que se abordará el análisis, construcción y resolución de problemas que tengan que ver con los triángulos, se propone ofrecerles a los estudiantes una herramienta desde una visión dinámica y participativa, mediante la aplicación de las tecnologías de la información y la comunicación (TIC). La investigación fue diseñada para estudiantes de grado séptimo. La experiencia se compone de un diseño curricular y un modelo pedagógico fundamentando en el constructivismo, y un referente tecnológico sobre la importancia del uso y aplicación de las TIC en la educación. La estrategia buscó conocer los triángulos mediante su clasificación, sus elementos y sus aplicaciones, de manera didáctica, a través del software educativo "El mundo de los triángulos". Con el software educativo se consiguió que los estudiantes de grado séptimo elevaran sus niveles de motivación y concentración, obteniendo así mayor disposición hacia la adquisición de nuevos conocimientos.
\end{abstract}

Palabras clave: herramienta pedagógica, tecnológico, triángulos, didáctica, educación, TIC.

1. Artículo de reflexión, con un enfoque cualitativo, resultado del trabajo para obtener el título de magíster, perteneciente al área de Sistemas, subárea de Informática, desarrollado en el grupo de investigación ANTA y financiado por la Universidad Cooperativa de Colombia de la ciudad de Bogotá (Colombia). Dirección Avenida Caracas No. 37-63, PBX: 3323565. Fecha de inicio: enero 2016, fecha de terminación: diciembre 2016.

2. Ingeniero de Sistemas, Universidad Cooperativa de Colombia. Magister en Informática Aplicada a la Educación Universidad Cooperativa de Colombia. Docente Investigador del grupo ANTA. Universidad Cooperativa de Colombia de la Ciudad de Bogotá - Colombia, Avenida Caracas n. ${ }^{\circ}$ 37-63, PBX: 33235 65. - Orcid: 0000-0002-3548-0775. Correo institucional: Fredys.simanca@campusucc.edu.co

3. Ingeniera de Sistemas Universidad Antonio Nariño, especialista en Docencia universitaria, especialista en Multimedia para la docencia, Magister en Informática Aplicada a la Educación, Universidad Cooperativa de Colombia, Coordinador grupo de investigación Espinfot, docente Universidad Distrital Francisco José de Caldas, Bogotá (Colombia). Dirección: Cra. 7 n. ${ }^{\circ}$ 40B - 53, Bogotá. - Orcid: 0000-0001-8869-7129. Correo institucional: aabucharp@udistrital.edu.co.

4. Ingeniero de Sistemas, Universidad Piloto. Magister en Informática Aplicada a la Educación, Universidad Cooperativa de Colombia. Docente Investigador del grupo ANTA. Universidad Cooperativa de Colombia de la ciudad de Bogotá (Colombia). Dirección: Avenida Caracas n. ${ }^{\circ}$ 37-63. PBX: 33235 65. Orcid: 0000-0001-7131-4427 Correo institucional: fabian.blanco@campusucc.edu.co.

5. Ingeniero de Sistemas, Universidad Cooperativa de Colombia. Magister en Informática Aplicada a la Educación, Universidad Cooperativa de Colombia. Docente investigador del grupo ANTA. Universidad Cooperativa de Colombia de la ciudad de Bogotá (Colombia). Dirección: Avenida Caracas n. ${ }^{\circ}$ 37-63, PBX: 33235 65. Orcid: 0000-0003-1367-9684. Correo institucional: pablo.carreno@campusucc.edu.co. 


\begin{abstract}
This work was born from the teaching of a work team of three institutions. After identifying the need to strengthen the teaching learning processes applying ICTThrough the development of technological tools as support for teaching practice, specifically in the analysis, construction and resolution of problems related to the triangle, should be offered to students from a dynamic and participatory vision through the application of information and communication technologies (ICT), as a pedagogical tool for the construction of knowledge. The research was designed for seventh grade students of IED Carlos Pizarro León Gómez educational institution located in the seventh location of Bosa Bogotá-Colombia; which is composed of a curricular design and a pedagogical model based on the proposal (constructivism) and a technological reference on the importance of the use and application of ICT in education. The strategy sought to make known the triangle through its classification, its elements and some applications in a didactic and attractive way, making the educational software "The world of triangles", will arouse interest in the technological innovation by the students, raising the levels of motivation and concentration, thus obtaining greater disposition towards the acquisition of new knowledge.
\end{abstract}

Keywords: pedagogical tool, technological, triangles, didactics, ICT education.

El uso de las TIC en los entornos educativos cada día es más generalizado, ya que la aplicación de nuevas tecnologías, combinadas con recursos multimedia, permite a los estudiantes más compresión y entendimiento de los triángulos, lo cual conlleva una exigencia mayor en los docentes para ser más creativos en la enseñanza (Abuchar, Simanca \& Cárdenas, 2013). "En los niños existe un impulso de hacer cosas, un instinto que solo puede explicarse como una necesidad permanente de experimentar, de indagar, de relacionar, como un potencial innato", que debe mantenerse e involucrarse en la práctica docente con los nuevos ambientes tecnológicos. Es por esa necesidad permanente de experimentar, que este artículo está enmarcado en la práctica docente, en este caso en particular en la enseñanza de la matemática en la educación básica, ya que, según Piedrahita (2007):

El objetivo central de la educación matemática y sus acciones, se basan en el compartir con los estudiantes una parte de la estructura teórica ya construida, utilizando para ello diferentes recursos y herramientas. Luego entonces es necesario en encontrar sentido a una parte del gran acumulado de significados validados en el campo matemático desde la teoría, y compartidos por el docente, de tal forma que sean utilizados por ellos en su vida diaria, en los ámbitos profesional y cotidiano no formalizado.

El estudio de la matemáticas tiene dos vertientes: las matemáticas puras y las matemáticas aplicadas; esta investigación se centra en la matemática pura, que se ocupa de los números, los vectores, los teoremas, el álgebra y la geometría, materia que es el punto de partida de este trabajo. Algunos maestros relacionan la geometría, principalmente, con temas como perímetros, áreas y volúmenes, limitándola solo a mediciones; para otros docentes la principal preocupación es dar a conocer las figuras o relaciones geométricas utilizando dibujos, el nombre de cada una y su definición, limitando el proceso de enseñanza-aprendizaje a una especie de glosario geométrico ilustrado (García \& López, 2008).

Es importante reflexionar sobre las razones para enseñar geometría. Si se tiene claro el por qué, se estará en condiciones de tomar decisiones acertadas acerca de la enseñanza. La razón principal se encuentra en el entorno inmediato, pues basta con mirar y descubrir que en él se encuentran muchas relaciones y conceptos geométricos, en tanto que la geometría modela el espacio que se observa, es decir, la geometría es la matemática del espacio (García \& López, 2008). No obstante que la presencia de la geometría en el entorno inmediato podría ser una razón suficiente para justificar su enseñanza y su aprendizaje, no es la única. La geometría ofrece a quien la aprende una oportunidad de emprender un viaje hacia formas superiores de pensamiento (García \& López).

En la actualidad, la sociedad está inmersa en el desarrollo tecnológico; el avance de las tecnologías de la información y la comunicación (TIC) han cambiado la forma de vida e impactado en todas las áreas del conocimiento. En el área educativa, las TIC han demostrado ser de apoyo tanto para los docentes como para los estudiantes. En este sentido, la implementación de la tecnología en la educación debe verse como una herramienta de apoyo, que no sustituye el papel del docente, sino que coadyuva para que el estudiante tenga más elementos (visuales y auditivos) para enriquecer el proceso de enseñanzaaprendizaje (Académica, 2013).

El uso de las TIC a través del software educativo, permitió que la apropiación y aprehensión de los conceptos 
relacionados con la enseñanza de los triángulos sean más didácticas e interactivas, logrando establecer relaciones espaciales y geométricas. Para Abrate y Pochulu (2005) una herramienta de apoyo a los procesos de enseñanza y aprendizaje de la matemática es el software educativo, el cual, si está bien elaborado y se hace un uso adecuado de él, puede mejorar notablemente el interés y la construcción de conocimiento matemático en los alumnos. No obstante, es necesario que todo docente conozca algunas normas y criterios para seleccionar un buen software de matemática, puesto que de ello dependerá que se fortalezca el aprendizaje de los estudiantes.

La población objeto de la investigación estuvo conformada por estudiantes de grado séptimo (701-703) de educación básica de la Institución Educativa Distrital Carlos Pizarro León Gómez, jornada mañana, que ofrece los niveles de preescolar, básica primaria, secundaria y media fortalecida dentro del programa de jornada completa.

Los autores presentan como estrategia de aprendizaje de los triángulos el desarrollo de un software que se ha titulado "El mundo de los triángulos", el cual facilita los procesos de compresión y entendimiento de la geometría y específicamente, la enseñanza de los triángulos. Este software lo utilizarán los estudiantes de grado séptimo jornada mañana de la IED Carlos Pizarro León Gómez de la ciudad de Bogotá, y su objetivo es identificar y conocer los triángulos de acuerdo a sus características, clasificación, elementos y aplicaciones de una manera didáctica, llamativa e interactiva para los estudiantes. El software está estructurado empleando recursos como videos, sonidos, conceptos teóricos, ejemplos y actividades. Acosta (2010) dice que con el uso de las TIC en el proceso enseñanza-aprendizaje y en particular en las matemáticas, las herramientas tecnológicas se han convertido en un elemento de motivación para los estudiantes, ya que ellos hacen parte de la generación de "nativos digitales".

La construcción del software educativo "El mundo de los triángulos", será uno de los primeros ambientes de interacción de los estudiantes con el aprendizaje mediado por las TIC, que permitirán en un futuro construir un ecosistema digital, es uno de los objetivos estratégicos del Ministerio de Tecnologías de la Información y las Comunicaciones -Mintic, con el fin de potenciar el desarrollo tecnológico del país, reducir la pobreza y alcanzar un mayor acceso y calidad de la educación. Entiéndase como ecosistema digital la articulación dinámica y sinérgica de diferentes comunidades digitales, que consisten en la interconexión, interrelación e interdependencia de recursos digitales (Cárdenas, Suárez \& Guerrero, 2014).

\section{Metodología}

La metodología empleada presenta elementos de investigación cuasiexperimental y comparativa. La investigación comparativa -que se funda en la comparación, en tanto actividad de la razón que pone en correspondencia unas realidades con otras para ver sus semejanzas y diferencias- tiene como objeto identificar las diferencias o semejanzas con respecto a la aparición de un evento en dos o más contextos, grupos o situaciones diferentes (Sierra, 1994). La comparación es posible porque existe una relación de analogía entre las diversas realidades (Aníbal, 2007).

Teniendo en cuenta los planteamientos de Sierra (1994) y Aníbal (2007), se propuso el siguiente interrogante: ¿qué progresos se evidencian en los procesos de enseñanza - aprendizaje de los triángulos en los estudiantes de grado séptimo del colegio IED Carlos Pizarro León Gómez mediante el uso del software "El mundo de los triángulos"?

La geometría se aplica en la realidad (en la vida cotidiana, la arquitectura, la pintura, la escultura, la astronomía, los deportes, la carpintería, la herrería, etcétera), y permite desarrollar en los alumnos su percepción del espacio, su capacidad de visualización y abstracción, su habilidad para elaborar conjeturas acerca de las relaciones geométricas en una figura o entre varias y su habilidad para argumentar al tratar de validar las conjeturas que hace (García \& López, 2008).

La aplicación de un criterio de clasificación para formar grupos permitió la comparación de estudiantes de grado séptimo (701-703), ambos con base en un evento para determinar si cada uno de los grupos se parecen o no según el esquema para una investigación comparativa (Hurtado, 2007). En la investigación comparativa el investigador trabaja con un evento o característica de estudio (del cual se derivan los criterios de comparación) y trata de identificar el comportamiento diferencial de dicho evento en varias situaciones, contextos, fenómenos o grupos.

\section{Características de la población objeto}

La población de esta investigación está en la comunidad académica del Centro Educativo Comunal en el barrio La Libertad de Bosa, que fue creado en el año de 1996, y que inicialmente funcionaba en un edificio de tres plantas con tres jornadas, para atender las necesidades educativas 
del sector (Colegio Carlos Pizarro León Gómez, 2009); esta institución, que es de carácter público y distrital, está ubicada en la calle 72 sur n. ${ }^{\circ} 100 \mathrm{~A}-51$, Ciudadela El Recreo, localidad séptima de Bosa, al suroccidente de la ciudad de Bogotá; es un colegio de los denominados megacolegios, cuenta con biblioteca, comedor, canchas de baloncesto y microfútbol, cuatro salas de informática, dos salas de profesores, cincuenta salones de clase.

En la actualidad presta servicio a más de tres mil estudiantes en dos jornadas. La institución toma el nombre de Carlos Pizarro León Gómez, personaje polémico, seminarista, estudiante de Derecho de la Universidad Javeriana y comprometido en buscar cambios y disminuir las desigualdades, militante del M19, candidato presidencial (Colegio Carlos Pizarro León Gómez, 2009).

En la institución educativa el área de matemáticas está integrada por ocho profesores (cuatro hombres y cuatro mujeres) con experiencia docente trabajando con niños, niñas y jóvenes; el $66 \%$ son licenciados en Matemáticas, el $17 \%$ son licenciados en Física y el $17 \%$ tienen otras licenciaturas.

La población objeto de investigación estuvo conformada por 82 estudiantes de grado séptimo (701-703, conformada por 43 hombres y 39 mujeres, con un promedio de edad entre 11 y 13 años; el $90 \%$ nacieron en la ciudad de Bogotá; en su mayoría pertenecen a EPS privadas; tienen necesidades educativas especiales, problemas de visión, alergias, migraña, rinitis, asma; se encuentran ubicados en los barrios El Recreo, Villas de Vizcaya, Alameda El Recreo, Ciudadela El Recreo, Brasil y Atalayas de la localidad séptima de Bosa; condición socioeconómica de estrato 2 ; el $73 \%$ de sus familias están integradas por el padre, la madre y los hermanos, el nivel de escolaridad en el padre es primaria, $22 \%$ y secundaria, $66 \%$; en la madre es $72 \%$, secundaria, y $17 \%$, universitario; el $83 \%$ de los estudiantes dicen tener como mínimo un computador en la casa y con acceso a internet, en su mayoría dedican entre 1 y 3 horas diarias a navegar en internet.

En cuanto a sus intereses académicos las asignaturas por las que manifiestan preferencia son tecnología y educación física, mientras que las asignaturas de las que menos gustan son matemática, español e inglés; su tiempo libre lo dedican a actividades lúdicas como bailar, jugar y patinar.

\section{Formulación de la prueba}

Para aplicar correctamente esta prueba se tuvieron en cuenta los requisitos que indican Colás y Buendía (1998):

Determinar de antemano los objetivos que se pretenden comprobar.

Tener presente el tipo de proceso mental que se pretende que realice el alumno.

La calidad de la prueba puede bajar si hay que contestar mucho en poco tiempo. Y si, por el contrario, el tiempo es excesivo, puede hacer su aparición el cansancio, impidiendo igualmente, que se haga una valoración justa del objetivo propuesto.

Estos autores también señalan:

- La utilización de pruebas escritas para comprobar el rendimiento académico de los alumnos es la forma más utilizada en todos los niveles de enseñanza.

- La selección del tipo de prueba y una correcta formulación de los ítems facilitará la labor de evaluación.

Para comprobar el rendimiento escolar se aplica la prueba objetiva, que a su vez, puede ser de múltiple elección de respuesta, en la que después de un enunciado en forma de pregunta o frase incompleta, se presentan varias respuestas entre las que el alumno debe elegir la que considere correcta (Peña, 2010).

\section{Instrumento de medición}

Teniendo en cuenta las necesidades observadas, se diseñan los siguientes instrumentos de recolección de información:

- Instrumento de medición o prueba de conocimiento aplicado a los estudiantes.

\section{- Encuesta para los estudiantes.}

La prueba de conocimiento está compuesta por preguntas de selección con única respuesta, las cuales están relacionadas con el triángulo, sus elementos, propiedades, el teorema de Pitágoras, clasificación según sus lados y sus ángulos. La encuesta consta de quince preguntas, cada una de las cuales pretende medir el nivel de conocimientos de los estudiantes acerca de los triángulos. Las preguntas propuestas en el instrumento de medición permiten determinar los conceptos previos que poseen los estudiantes sobre el tema. 


\section{Procedimiento}

Los estudiantes del grado 703, que integran el grupo de control, en el desarrollo de su clase de triángulos utilizaron la cartilla relacionada con el software "El mundo de los triángulos", en la que se encuentran: palabras claves, objetivos, referentes teóricos, gráficos, ilustraciones, ejemplos, pasatiempos y actividades complementarias sobre cada uno de los temas propuestos en la unidad didáctica. Esta actividad estuvo orientada por el docente Fredys Simanca. Durante el desarrollo de la clase de triángulos, se estudiaron conceptos geométricos como las propiedades de los triángulos, los elementos del triángulo (altura, lados, ángulos, vértices, mediatriz, bisectriz, incentro, circuncentro, etcétera), la clasificación de los triángulos según sus lados (equilátero, isósceles, escaleno) y según sus ángulos (acutángulo, rectángulo, obtusángulo) y el teorema de Pitágoras.

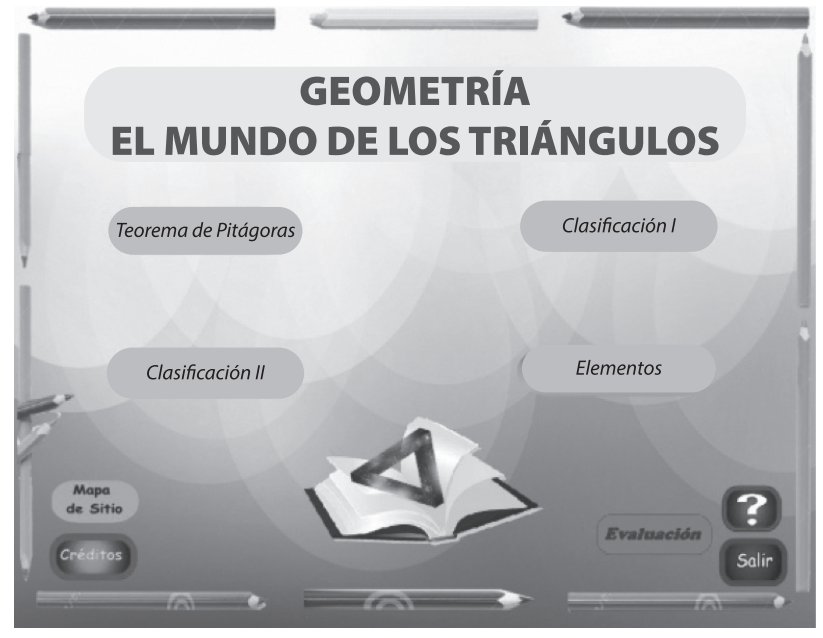

Figura 1.

Software "Elmundo de los triángulos"

Fuente:Autores

Para determinar y definir el impacto de la propuesta, los estudiantes del grado 701, que conforman el grupo experimental, son quienes van a utilizar el software "El mundo de los triángulos", cuyo diseño permite al estudiante aprender de manera autónoma mediante la interacción con el programa. Dicho software, que está construido didácticamente para que los estudiantes adquieran conceptos relacionados con la teoría de los triángulos, se compone de videos, tutoriales, referentes teóricos, palabras claves, objetivos, ejemplos, pasatiempos y retos que deben ir resolviendo durante su ejecución.

El objetivo central de la propuesta fue aprender el concepto de los triángulos, sus características y elementos, así como su clasificación teniendo en cuenta sus lados y sus ángulos, y el teorema de Pitágoras como una aplicación del triángulo rectángulo. Esta propuesta está acompañada del elemento necesario para el desarrollo y estructuración de un curso en aula virtual según Abuchar y Cárdenas (2012) ya que permite afianzar y potencializar los conocimientos desarrollados mediante el manejo interactivo del software a través de actividades de refuerzo, videos y evaluación sobre el tema.

Como punto de innovación se implementó la realidad aumentada como un instrumento que permitió que los estudiantes comprendieran de manera gráfica, y acercándose a la realidad, las clases de triángulos, sus elementos, propiedades, aplicaciones y algunos ejemplos. Se pretendió romper con la monotonía de las clases mediante el uso de las herramientas mencionadas, y se innovó y potencializó la temática con la implementación de las TIC en la educación, generando espacios de creatividad en el estudiante.

Se optó por una mediación tecnológica en la educación como es la realidad aumentada, toda vez que esta es una técnica de apoyo fundamental, ya que permite tener al alcance de una pantalla variedad de contenido académico que permite reforzar los conocimientos motivando a la participación, habilitando destrezas y desarrollando esquemas mentales propios del individuo por medio del uso de la tecnología (Saguay, 2015).

\section{Resultados}

En las siguientes tablas y gráficos se puede analizar el nivel del aprendizaje en el que se encuentran los estudiantes del grado 7 del grupo experimental de la Institución Educativa Distrital Carlos Pizarro León Gómez. Los datos se obtuvieron al aplicar el instrumento de medición.

En la Figura 2 se puede observar que hubo un avance significativo en los estudiantes del grupo experimental de la Institución Educativa Distrital Carlos Pizarro León Gómez; estos datos se obtuvieron al aplicar el instrumento de medición pretest y postest. Sin embargo, es importante destacar que en las preguntas 3, 4 y 15 los resultados fueron inferiores, esto indica que los conceptos suma de ángulos internos de un triángulo y ángulo recto, no fueron claros.

En la Figura 3 se puede observar que hubo un avance significativo, aunque no en la misma proporción del grupo experimental, pues los porcentajes de avance fueron inferiores y además las preguntas 3,4 y 15 muestran resultados negativos, lo que indica una dificultad en la apropiación de los conceptos de suma 
de ángulos internos de un triángulo y ángulo recto; los resultados se obtuvieron al aplicar el instrumento de medición pretest y postest.

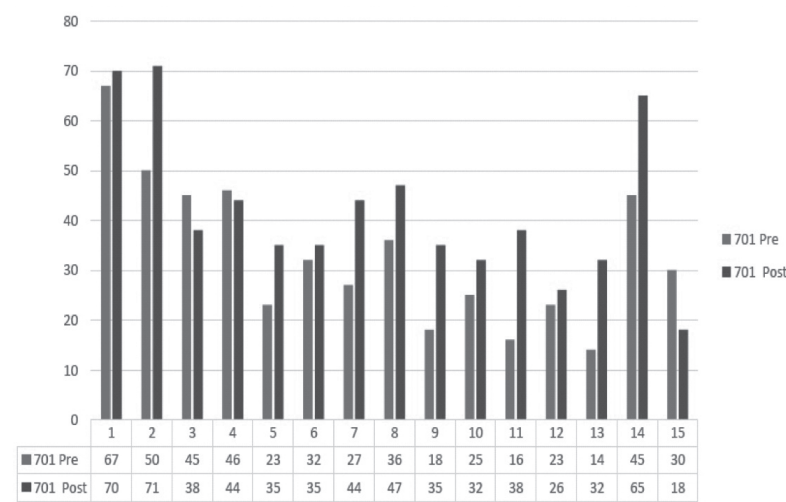

Figura 2.

Análisis comparativo de los datos del grupo experimental instrumento de medición pretest

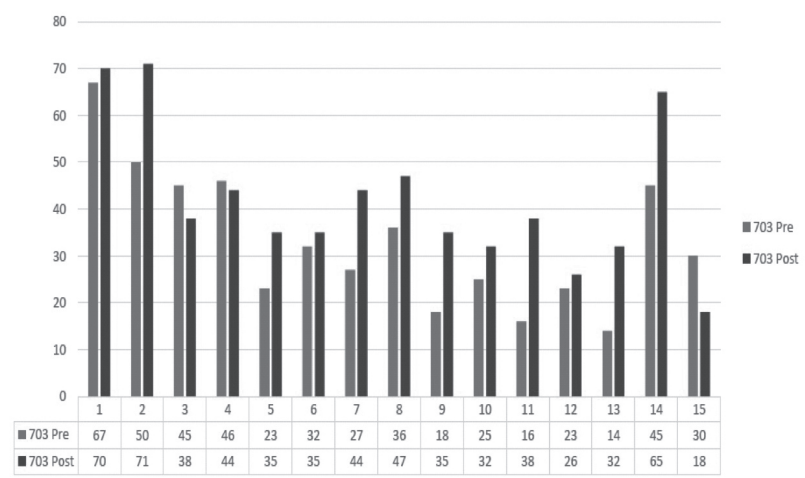

Figura 3.

Análisis comparativo de los datos del grupo control instrumento de medición pretestypostest

Al realizar el análisis DOFA en el grupo experimental se evidencian grandes fortalezas en el proceso de investigación; es importante destacar que la institución cuenta con equipos de computación actualizados, una red de internet, aulas especializadas en áreas del conocimiento como la matemática y un grupo de profesores que están en constante actualización y además cuentan con una amplia experiencia en la labor docente. Entre las oportunidades es importante resaltar la proyección que la institución muestra hacia la incorporación de las TIC en el proceso enseñanzaaprendizaje.

\section{Análisis DOFA del grupo experimental}

\begin{tabular}{|c|c|c|}
\hline $\begin{array}{l}\text { FACTORES } \\
\text { EXTERNOS }\end{array}$ & $\begin{array}{l}\quad \text { Lista de Fortalezas } \\
\text { F1 Equipos de computación } \\
\text { F2 Internet } \\
\text { F3 Material humano } \\
\text { F4 Aulas digitales } \\
\text { F5 Dominio del concepto de } \\
\text { triángulo y ángulo recto } \\
\text { F7 E1 método de enseñanza } \\
\text { F8 E1 currículo } \\
\text { F9 Experiencia docente }\end{array}$ & \begin{tabular}{l}
\multicolumn{1}{c}{ Lista de Debilidades } \\
D1 Número de estudiantes \\
D2 Conexión a internet \\
D3 La familia \\
D4 Formación académica \\
D5 Método tradicional \\
D5 Poco dominio en temas \\
como: propiedades del \\
triángulo, clasificación, \\
triángulo escaleno, elementos \\
del triángulo, triángulo \\
rectángulo, teorema de \\
Pitágoras y ángulos
\end{tabular} \\
\hline $\begin{array}{l}\text { Lista de Oportunidades } \\
\text { O1 Acceso a la educación } \\
\text { O2 software educativo } \\
\text { O3 Material humano } \\
\text { O4 Acceso a la tecnologia }\end{array}$ & $\begin{array}{l}\text { Estrategia para maximizar } \\
\text { tanto las } \mathbf{F} \text { como las } O \\
\text { Formación y capacitación } \\
\text { docente en el uso de las TIC }\end{array}$ & $\begin{array}{l}\text { Estrategia para minimizar } \\
\text { las D y maximizar las } 0 \\
\begin{array}{l}\text { Fortalecer el curriculo } \\
\text { educativo en el área de } \\
\text { matemáticas }\end{array}\end{array}$ \\
\hline $\begin{array}{l}\text { Lista de Amenazas } \\
\text { A1 Entomo social } \\
\text { A2 Redes sociales } \\
\text { A3 Uso de la tecnologia } \\
\text { A4 Deserción escolar } \\
\text { A5 Grupos sociales } \\
\text { A6 Desintegración de las } \\
\text { familias }\end{array}$ & $\begin{array}{l}\text { Estrategia para maximizar } \\
\text { las F y minimizar las A } \\
\text { Formación en manejo de redes } \\
\text { sociales }\end{array}$ & $\begin{array}{l}\text { Estrategia para minimizar } \\
\text { tanto las D como las A }\end{array}$ \\
\hline
\end{tabular}

Figura 4.

Análisis DOFA del grupo experimental

\section{Análisis DOFA del grupo de control}

\begin{tabular}{|c|c|c|}
\hline $\begin{array}{l}\text { FACTORES } \\
\text { INTERNOS }\end{array}$ & $\begin{array}{l}\quad \text { Lista de Fortalezas } \\
\text { F1 Crecimiento personal } \\
\text { F2 Estudiantes y profesores } \\
\text { F3 Material humano } \\
\text { F4 Espacios de trabajo } \\
\text { F5 Conocimientos previos } \\
\text { F6 Motivación } \\
\text { F7 El curriculo } \\
\text { F8 Material didáctico } \\
\text { F9 Experiencia docente }\end{array}$ & $\begin{array}{l}\text { Lista de Debilidades } \\
\text { D1 Número de estudiantes } \\
\text { D2 Recursos } \\
\text { D3 La familia } \\
\text { D4 E1 Tiempo } \\
\text { D5 Contexto social }\end{array}$ \\
\hline $\begin{array}{l}\text { Lista de Oportunidades } \\
\text { O1 Participar del proyecto } \\
\text { O2 Materiales de trabajo } \\
\text { 03 Ampliar conocimientos } \\
\text { O4 Extensión del proyecto } \\
\text { O5 Aplicar esta estrategia en } \\
\text { otras áreas } \\
\text { O6 Identificación de puntos a } \\
\text { mejorar }\end{array}$ & $\begin{array}{l}\text { Aplicar nuevos modelos de } \\
\text { enseñanza. }\end{array}$ & $\begin{array}{l}\text { Estrategia para minimizar } \\
\text { las D y maximizar las } 0 \\
\text { Seleccionar la temática que se } \\
\text { debe implementar en los } \\
\text { estudiantes, sin olvidar los } \\
\text { estándares del ministerio de } \\
\text { educación y las competencias } \\
\text { que se deben alcanzar. }\end{array}$ \\
\hline $\begin{array}{l}\quad \text { Lista de Amenazas } \\
\text { A1 Entomo social } \\
\text { A2 Interés en el proyecto } \\
\text { A3 Apoyo de la institución } \\
\text { A4 Deserción escolar } \\
\text { A6 Apoyo familiar } \\
\text { A7 Espacios de trabajo }\end{array}$ & $\begin{array}{l}\text { Estrategia para maximizar } \\
\text { las F y minimizar las A } \\
\text { Charlas orientadoras enfocadas } \\
\text { en la familia, entorno social y } \\
\text { valores. }\end{array}$ & $\begin{array}{l}\text { Estrategia para minimizar } \\
\text { tanto las D como las A } \\
\text { Revisión del modelo educativo }\end{array}$ \\
\hline
\end{tabular}

Figura 5.

Análisis DOFA del grupo de control 


\section{Variables de estudio}

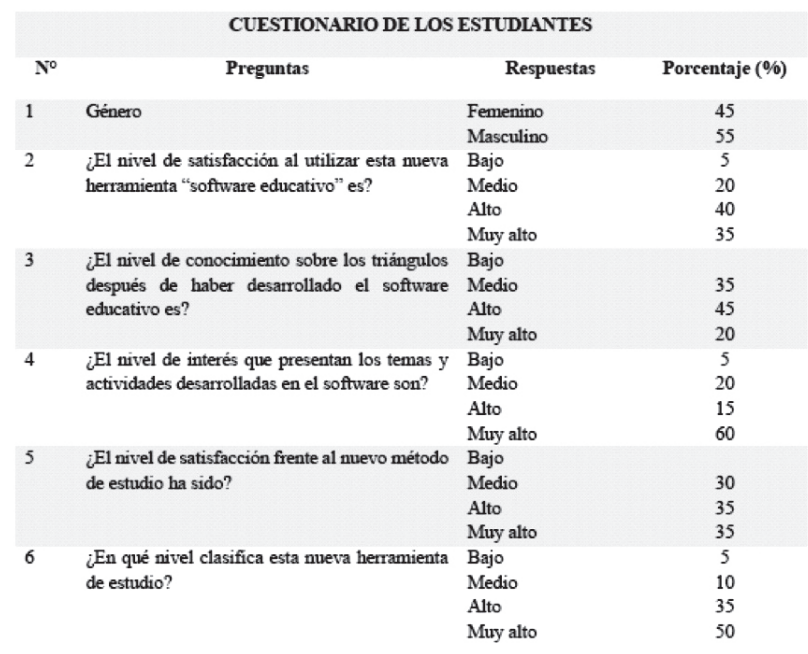

Figura 6.

Resultado obtenido dela encuesta a los estudiantes

El cuestionario consta de seis preguntas. Una para establecer el género del estudiante, las cuatro restantes son preguntas de información, y finalmente, una pregunta abierta que los estudiantes contestaron libremente.

Durante la aplicación del software educativo se observó que la mayoría de los estudiantes tienen gran interés y expectativa acerca de las actividades propuestas y la implementación de una estrategia metodológica novedosa y diferente a lo tradicional, que haga la clase entretenida y eficiente; el tema de los triángulos fue bien acogido y se desarrolló en su totalidad cada uno de los objetos virtuales de aprendizaje, OVA.

\section{Conclusiones}

Para dar respuesta a la pregunta problema planteada en el trabajo de investigación y teniendo en cuenta los datos obtenidos en el pretest y el postest de los grupos objeto de estudio, se puede determinar que el nivel de aprendizaje de los estudiantes en cuanto a los elementos del triángulo, su clasificación y algunas aplicaciones tuvo un avance significativo. Pasaron de tener una media de $33,1 \%$ a una media del $42 \%$, lo que indica que hubo un progreso en el proceso de enseñanza - aprendizaje del $9 \%$. Los estudiantes terminaron el proceso con un nivel alto en cuanto a la definición del triángulo, y con un nivel medio en la identificación de los elementos del triángulo, su clasificación e identificación de algunas aplicaciones.

El uso de las TIC como herramienta tecnológica permitió que los estudiantes del grupo experimental fueran adquiriendo, paso a paso y a ritmos diferentes, el conocimiento sobre los elementos y puntos notables del triángulo, identificaran y clasificaran los triángulos teniendo en cuenta sus lados y sus ángulos y, finalmente, reconocieran en el teorema de Pitágoras un recurso para resolver situaciones problemáticas relacionadas con los triángulos.

Es así que, el software educativo está dirigido a una población heterogénea en cuanto a edades, niveles cognitivos y capacidades diferentes. La metodología desarrollada, que consistió en crear un software educativo que se implementó por parejas, favoreció la comunicación entre los estudiantes de grado séptimo, debido a las múltiples y variadas actividades propuestas. El desarrollo del software permitió determinar que los estudiantes se sienten protagonistas de su propio aprendizaje, hecho que facilita el proceso de aprender a aprender y permite, a su vez, que los alumnos se acerquen de una manera diferente -a través de las TIC- $\mathrm{a}$ la geometría.

Para la ejecución y la implementación del software educativo "El mundo de los triángulos", los estudiantes del grupo experimental tuvieron la posibilidad de acceder a aulas digitales o autosuficientes, que cuentan con los recursos necesarios para posibilitar al estudiantado el acceso a la información y la comunicación que proporciona la red y los sistemas de presentación multimedia disponibles. Con las aulas digitales, se posibilitó a los estudiantes la mejor calidad posible en cuanto al desarrollo del software educativo y se les permitió acceder a la información de una manera fácil y dinámica.

Los maestros del área de matemáticas de la IED Carlos Pizarro León Gómez quieren incorporar los recursos tecnológicos en el aula de clase, dando respuesta a los retos que plantea la sociedad actual. Sin embargo, la incorporación de las TIC en el proceso de enseñanzaaprendizaje no solo supone la dotación de computadores y el acceso a internet, sino que implica compromiso y capacitación por parte de los maestros y que los estudiantes le den buen uso a las redes sociales $y$, en general, al recurso tecnológico.

Es necesario plantear actividades para aplicar los conceptos desarrollados. En este momento los estudiantes muestran que han asimilado el concepto de triángulo, sus propiedades y su clasificación, aunque lo expresan con un lenguaje poco preciso. Los alumnos aplican sus conocimientos a los problemas teniendo claro cómo deben ser resueltos a través del software, lo que indica que este es, sin lugar a dudas, una ayuda importante. En términos generales, los alumnos 
que participaron del estudio presentan capacidades cognitivas diferentes entre sí, lo cual significa que cada uno logró interiorizar el aprendizaje de manera significativa.

El método tradicional de aprendizaje, aunque muestra avances, no genera un cambio significativo en el nivel de aprendizaje de los estudiantes. Por su parte, la implementación del software educativo "El mundo de los triángulos" como una estrategia didáctica, despertó en los estudiantes el interés ante la novedad tecnológica, generando curiosidad y elevando los niveles de motivación y concentración, obteniéndose así mayor disposición hacia la adquisición de los nuevos conocimientos.

Al comparar los resultados obtenidos en los instrumentos pretest y postest por los grupos experimental y de control se pudo evidenciar que después de la aplicación del software educativo "El mundo de los triángulos" los estudiantes del grupo experimental obtuvieron un avance significativo del $9 \%$, mientras que el grupo control mejoró un 3,5\%.

\section{Referencias}

Abrate, R. \& Pochulu, M. (2005). El software educativo en la enseñanza y aprendizaje de la matemática: fortalezas, oportunidades, debilidades y amenazas.

Abuchar, A. \& Cárdenas, B. (2012). Aproximación modelo para el diseño de cursos virtuales.

Abuchar, A., Simanca, F. \& Cárdenas, B. (2013). Naturaleza creativa. Tecnología, Investigación y Academia, 1(1), 64-75.

Académica. (2013). Importancia del uso de las TIC en la educación. Recuperado de http://www.academica.mx/ blogs/importancia-del-uso-las-tic-en-la-educacion

Acosta, E. (2010). Enseñando transformaciones geométricas con software de geometría dinámica. Encuentro Colombiano de Matemática Educativa.

Aníbal, P. (2007). El método comparativo: fundamentos y desarrollos recientes. Pittsburgh: Departamento de Ciencias Políticas universidad de Pittsburgh.

Cárdenas, J., Suárez, J. \& Guerrero, C. (2014). Ecosistema digital académico: una comunidad digital soportada en TIC para las instituciones de educación superior. I+D Revista de Investigaciones, 4(2), 6-14.

Colás, P., Buendía, L. \& Hernández, F. (1998). Métodos de investigación.
Colegio Carlos Pizarro León Gómez. (2009). Historia. Recuperado de http://colegiocarlospizarro.blogspot.com.co/2009/04/ historia.html

Hurtado, J. (2007). Tipos de Metodología de la Investigación - Cómo Hacer una Metodología. Recuperado de http:// aprenderlyx.com/tipos-de-metodologia-de-investigacion.

García, S. \& López, O. (2008). La enseñanza de la Geometría.

Peña, A. (2010). Enseñanza de la Geometría con TIC en educación secundaria obligatoria. Madrid: Universidad Nacional de Educación a Distancia.

Piedrahita, W. (2007). Geometría y Desarrollo Humano. Educación Hoy, Confederación Interamericana de Educacion, 67-82.

Saguay, L. (2015). Desarrollo de material didáctico basado en realidad aumentada para la enseñanza de geometría en educación básica media. (Tesis Magister en Tecnologías para Gestión y Práctica Docente). Pontificia Universidad Católica del Ecuador.

Sierra, R. (1994). Técnicas de investigación Social Teoría y ejercicios. McGraw Hill. 